\title{
Emergence of extended spectrum beta-lactamases-producing strains belonging to cefotaxime-M-1 class from intensive care units patients and environmental surfaces in Pakistan
}

Aqsa Ashraf Bukhari, Muhammad Imran Arshad, Shahbaz Raza, Mariya Azam, Sajjad-ur-Rahman and Mashkoor Mohsin

\author{
Institute of Microbiology, University of Agriculture, Faisalabad 38040, Pakistan. \\ Corresponding author: Mashkoor Mohsin, e-mail: mashkoormohsin@uaf.edu.pk, \\ AAB: bukhari_aqsa@yahoo.com, MIA: drimranarshad@yahoo.com, SR: shahbaz.invincible3182@gmail.com, \\ MA: mazam.87@gmail.com, SUR: sajjadur@uaf.edu.pk, MM: mashkoormohsin@uaf.edu.pk \\ Received: 20-08-2016, Accepted: 01-11-2016, Published online: 22-11-2016
}

doi: 10.14202/IJOH.2016.69-74 How to cite this article: Bukhari AA, Arshad MI, Raza S, Azam M, Sajjad-ur-Rahman, Mohsin M. Emergence of extended spectrum beta-lactamases-producing strains belonging to cefotaxime-M-1 class from intensive care units patients and environmental surfaces in Pakistan. Int J One Health 2016;2:69-74.

\begin{abstract}
Aim: The emergence of multidrug-resistant (MDR) bacteria is the most dangerous threat for the treatment of infectious diseases. The aim of this study was to detect and characterize extended spectrum beta-lactamases (ESBLs) and carbapenemaseproducing Klebsiella pneumoniae and Escherichia coli among patients and environment of intensive care units (ICUs) of three tertiary care hospitals in Pakistan.

Materials and Methods: A total of 82 samples from ICU's patients and inanimate environment (injection trays, wash basins, door handles, hand swabs of professionals, and ICU fridges) were screened for ESBL by culturing on CHROMagar-ESBL. ESBL and carbapenemases production were confirmed by double disc synergy test and modified Hodge's test, respectively. Polymerase chain reaction was used to detect ESBL encoding genes bla cefotaxime (CTX-M), blaCTX-M-1, blaCTX-M-2, blaCTX-M-9, blaTEM, blaSHV and carbapenemase genes blaKPC, bla New Delhi metallo-beta-lactamase-1, blaOXA-48 and blaVIM.

Results: Overall, ESBL production was found high 30/82 (36.5\%) among isolates of which $15.8 \%$ K. pneumoniae and $20.7 \%$ E. coli were identified. All the K. pneumoniae and majority of E. coli isolates were MDR, i.e., resistance to three or more antimicrobial categories. Molecular characterization showed the blaCTX-M-1 as the predominant genotype found in $17 / 21(80 \%)$ of the isolates. None of the strains was found positive for carbapenemase-encoding genes.

Conclusion: In conclusion, this study demonstrates the emergence of MDR ESBL producing strains among ICU patients and hospital environment, posing a serious threat for the control of nosocomial infections.
\end{abstract}

Keywords: antimicrobial resistance, Escherichia coli, extended spectrum beta lactamase, Klebsiella pneumoniae, nosocomial infections.

\section{Introduction}

The antimicrobial resistance poses a serious public health problem and immense economic losses worldwide. The irrational use of antimicrobial agents in human medicine has led to rapid increase in bacterial resistance toward antimicrobial drugs [1]. Resistance to beta-lactam antibiotics, especially to $3^{\text {rd }}$ generation cephalosporins has become a global dilemma in infection control. Extended spectrum beta-lactamases (ESBLs) are a group of enzymes produced mostly by Gram-negative bacilli which are responsible for resistance to all $\beta$-lactam antibiotics except cephamycins and carbapenems [2]. Hospitalacquired infections (HAIs) or nosocomial infections are a source of diseases that may pose a threat to public health. The occurrence of infection to patients

Copyright: Bukhari, et al. This article is an open access article distributed under the terms of the Creative Commons Attribution 4.0 International License (http://creativecommons.org/licenses/ by/4.0/), which permits unrestricted use, distribution, and reproduction in any medium, provided you give appropriate credit to the original author(s) and the source, provide a link to the Creative Commons license, and indicate if changes were made. The Creative Commons Public Domain Dedication waiver (http:// creativecommons.org/ publicdomain/zero/1.0/) applies to the data made available in this article, unless otherwise stated. within 48-h of hospital stay or a month after any invasive procedure is known as nosocomial infections [3]. ESBL producing Enterobacteriaceae, especially Klebsiella pneumoniae and Escherichia coli are recognized globally as major causes of nosocomial and community-acquired infections [4]. Alone E. coli causes an estimated 120 million cases of community-acquired urinary tract infections (UTIs) worldwide each year [5]. Other complications include neonatal meningitis, pneumonia, and surgical site infections. Furthermore, in the last two decades, $K$. pneumoniae has emerged as an important cause of HAIs, especially among patients in the neonatal intensive care units (ICUs) with up to $70 \%$ mortality rates [6]. Person to person transmission has been known as the most frequent route of transmission of ESBL producing bacteria. Beside patients, environmental surfaces in hospitals could also be source of transmission of antimicrobial-resistant bacteria [7].

Resistance to $3^{\text {rd }}$ generation cephalosporin is predominantly mediated by plasmid encoded ESBL enzymes which have ability to hydrolyze $\beta$-lactam ring in these drugs [8]. Among the various genotypes of ESBLs, the most common are the SHV, TEM, and 
cefotaxime (CTX-M) types [7]. However, CTX-M type beta-lactamases are considered as the most important ESBL enzyme family [9]. Widespread dissemination of CTX-M in human hospital and community settings and its association with multidrug-resistance (MDR) has become a menace to global health [10]. More than 50 CTX-M types have been identified and are subdivided into five groups on the basis of amino acid differences (CTX-M-1, CTX-M-2, CTX-M-8, CTXM-9, and CTX-M-25) [11]. ESBL of CTX-M-1 and CTX-M-9 groups were the most common in Asia as well as worldwide [12]. Carbapenems were considered as the "last choice" of antibiotics for the treatment of nosocomial infections but recent reports on resistance to carbapenems due to the production of carbapenemase enzyme raised alarm in the medical community [13]. Among the most important carabepenemases are New Delhi metallo-beta-lactamase-1 (NDM-1) enzymes, which were originally reported from India and Pakistan [14]. Among other clinically important carbapenemases are KPC, VIM, and OXA48 types [15].

There is a paucity of information on the prevalence and molecular classes of ESBL and carbapenemase in hospital settings in Pakistan. Therefore, this study was designed to investigate the prevalence of ESBLs in clinical samples as well as inanimate environment of ICUs of different hospitals by phenotypic and molecular methods.

\section{Materials and Methods}

\section{Ethical approval}

The study was conducted in compliance with local Institutional Bioethics Committee (IBC), University of Agriculture, Faisalabad, Pakistan and Punjab Medical College, Faisalabad, Pakistan.

\section{Sample collection}

In the period from January to June 2015, sampling was executed at ICUs of three different tertiary care hospitals (Allied Hospital, DHQ Hospital, and Social Security Hospital) located in Faisalabad, Pakistan. An informed consent from the patients was obtained before including them in the study. Special attention was given to ICUs associated patients and environment. A total of 82 samples of which $n=64$ were isolated from wound, throat, and urine of hospitalized patients and $n=18$ from invasive devices, (urinary catheters, ventilators, oxygen masks, central venous catheter), injection tray, wash basins, door handles, hand swabs of professionals, ICU fridge/ dustbins in the ICU wards. The study was conducted in compliance with Institutional Bioethics Committee of University of Agriculture, Faisalabad, Pakistan.

Isolation of ESBL producing $K$. pneumoniae and $E$. coli All the 82 samples were plated directly on CHROMagar ESBL (CHROMagar, Paris, France) for presumptive detection of ESBL producing $K$. pneumoniae and E. coli. Biochemical identification of $K$. pneumoniae and $E$. coli was done by RapID ONE System (Remel, UK) according to the manufacturer's instructions.

\section{Antimicrobial susceptibility testing}

Antimicrobial susceptibility of presumptive ESBL producing $K$. pneumonaie and E. coli isolates was performed on Muller-Hinton agar (Oxoid, UK) by disc diffusion method following the CLSI criteria (16) with $30 \mu \mathrm{g}$ each of the third generation cephalosporins; ceftazidime (CAZ), CTX, and ceftriaxone (CRO). In addition, antimicrobial susceptibility of following antimicrobials was tested: Ampicillin (AMP), amikacin (AK) ciprofloxacin (CIP), tigecycline (TGC), chloramphenicol (C), nitrofurantoin (F), imipenem (IMP), and ertapenem (ETP). E. coli ATCC 25922 was used for quality control. MDR was defined as strains showing phenotypic resistance to at least three antimicrobial drugs belonging to three different classes.

\section{Phenotypic confirmation of ESBL}

All the K. pneumonaie and E. coli isolates were phenotypically confirmed for ESBL production using double disc synergy test (DDST) [16]. Briefly, a cefotaxime disc $(30 \mu \mathrm{g})$ was placed $20 \mathrm{~mm}$ away from a disc containing cefotaxime-clavulanic acid $(30 / 10 \mu \mathrm{g})$. When the inhibition zone between at least one of the combination discs and its corresponding single antibiotic disc differed by $\geq 5 \mathrm{~mm}$, the strain was identified as an ESBL producer.

\section{Phenotypic detection of carbapenem resistance}

For the phenotypic detection of carbapenems resistance, modified Hodge test (MHT) involving distorted carbapenem inhibition zones was performed [17]. Briefly, E. coli ATCC 25,922 was streaked as a lawn onto a Mueller Hinton agar plate. A $10 \mu \mathrm{g}$ IMP and/or ETP disc was placed in the center of the plate. Test isolate was streaked in a straight line from the edge of the disc to the edge of the plate. The plate was incubated overnight at $37^{\circ} \mathrm{C}$ for $18 \mathrm{~h}$. A cloverleaf type indentation at the intersection of the test organism within the zone of inhibition of the disc showed MHT positive.

\section{DNA extraction and quantification}

Total genomic DNA was extracted from the overnight enriched culture in brain heart infusion broth using the phenol-chloroform method [18]. The concentration and purity of the purified DNA were determined by measuring the optical density using Nanodrop (Thermo Fisher Scientific, MA). For all the polymerase chain reaction (PCR) reactions $50 \mathrm{ng} / \mu \mathrm{l}$ concentration was used as template.

\section{Molecular detection of ESBL and carbapenemase-en- coding genes}

The $\beta$-lactamase genes blaTEM, blaSHV, and blaCTX-M were investigated with PCR using previously described primers [19-21]. The three important subgroups of CTX-M-1, CTX-M-2 and 
CTX-M-9 were amplified using PCR primers and conditions as previously described [22]. For the detection of carbapenemase genes, PCR was used to target genes blaNDM-1 [23], blaOXA-48, blaKPC, and blaVIM [24]. Primer sequence, gene targeted, amplified product size, and annealing conditions are listed in Table-1.

\section{Results}

Prevalence of ESBL producing $K$. pneumoniae and $E$. coli in ICUs and associated environment

Out of 82 samples collected samples, ESBL production was phenotypically identified in $30(36.6 \%)$ isolates based on growth on CHROMagar-ESBL plates and antimicrobial susceptibility against cephalosporins. Overall, 13/82 (15.8\%) ESBL producing K. pneumoniae and $17 / 82(20.7 \%)$ ESBL producing $E$. coli isolates were identified. Out of ICU admitted patients, the highest number of ESBL isolates were found in throat followed by urine and wound. Furthermore, high percentage of ESBL isolates (33\%) was also found in samples from hospital environment (Table-2).

\section{Antimicrobial susceptibility pattern and ESBL confirmation}

Antimicrobial susceptibility results showed that all the 13/13 (100\%) K. pneumoniae and 15/17 (88.2\%)
E. coli isolates were MDR. Both $K$. pneumoniae and E. coli exhibited $100 \%$ resistance to CAZ, CRO, cefotaxime, AMP, and 100\% sensitivity to IMP. Among others, K. pneumoniae showed the highest resistance against ETP (76.9\%) followed by chloramphenicol (61.5\%), AK (46\%), CIP (38.4\%), TGC (38.4\%) and nitrofurantoin (23\%). In the case of E. coli, resistance was seen highest against nitrofurantoin $(58.5 \%)$ followed by ETP (41\%), AK (35.2\%), CIP (23\%), chloramphenicol (23\%), and TGC (5\%) (Figure-1). ESBL production among all presumptive isolates was further confirmed with DDST.

\section{ESBL isolates with positive carbapenemase resistance}

ESBL producing $K$. pneumoniae and $E$. coli isolates that were not susceptible to ETP were further analyzed for MHT. All the ETP resistant strains showed positive MHT test with characteristic cloverleaf-like indentation around ETP disc (Figure-2). However, none of the isolates was MHT positive with IMP.

\section{Molecular characterization of ESBL and carbapene- mase-encoding genes}

Of the 30 ESBL isolates, $23(76.6 \%)(13$ E. coli and $10 \mathrm{~K}$. pneumoniae) were positive for one of more beta-lactamases (bla) genes. Of these 23 isolates, $19(82 \%)$ carried blaCTX and 7 (30\%) harbored

Table-1: List of primers used for molecular characterization of ESBL.

\begin{tabular}{|c|c|c|c|c|c|}
\hline Target gene & Primer name & Sequence & $\begin{array}{l}\text { Product } \\
\text { size (bp) }\end{array}$ & $\begin{array}{c}\text { Annealing } \\
\text { temperature }\left({ }^{\circ} \mathrm{C}\right)\end{array}$ & References \\
\hline blaCTX-M & $\begin{array}{l}\text { blaCTX-M (F) } \\
\text { blaCTX-M (R) }\end{array}$ & $\begin{array}{l}\text { 5'SCS ATG TGC AGY ACC AGT'3 } \\
\text { 5'ACC AGA AYV AGC GGB GC'3 }\end{array}$ & 585 & 54 & [21] \\
\hline blaCTX-M-1 & $\begin{array}{l}\text { blaCTX-M-1 (F) } \\
\text { blaCTX-M-1 (R) }\end{array}$ & $\begin{array}{l}\text { 5'GCG TGA TAC CAC TTC ACC TC'3 } \\
\text { 5'TGA AGT AAG TGA CCA GAA TC'3 }\end{array}$ & 260 & 55 & [22] \\
\hline blaCTX-M-2 & $\begin{array}{l}\text { blaCTX-M-2 (F) } \\
\text { blaCTX-M-2 (R) }\end{array}$ & $\begin{array}{l}\text { 5'TGA TAC CAC CAC GCC GCT C'3 } \\
\text { 5'TAT TGC ATC AGA AAC CGT GGG'3 }\end{array}$ & 341 & 66 & {$[22]$} \\
\hline blaCTX-M-9 & $\begin{array}{l}\text { blaCTX-M-9 (F) } \\
\text { blaCTX-M-9 (R) }\end{array}$ & $\begin{array}{l}\text { 5'ATC AAG CCT GCC GAT CTG GTT A'3 } \\
\text { 5'GTA AGC TGA CGC AAC GTC TGC'3 }\end{array}$ & 293 & 62 & [22] \\
\hline blaTEM & 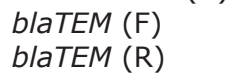 & $\begin{array}{l}\text { 5'ATG AGT ATT CAA CAT TTC CG'3 } \\
\text { 5'TT AAT CAG TGA GGC ACC TAT'3 }\end{array}$ & 867 & 53 & [19] \\
\hline blaSHV & $\begin{array}{l}\text { blaSHV (F) } \\
\text { blaSHV (R) }\end{array}$ & $\begin{array}{l}\text { 5'GGG TTA TTC TTA TाT GTC GC'3 } \\
\text { 5'TTA GCG TTG CCA GTG CTC'3 }\end{array}$ & 930 & 59 & [20] \\
\hline blaVIM & $\begin{array}{l}\operatorname{VIM}(F) \\
\operatorname{VIM}(R)\end{array}$ & $\begin{array}{l}\text { 5'AGT GGT GAG TAT CCG ACA G'3 } \\
\text { 5'ATG AAA GTG CGT GGA GAC'3 }\end{array}$ & 261 & 58 & [24] \\
\hline blaOXA-48 & $\begin{array}{l}\text { OXA-48 (F) } \\
\text { OXA-48 (R) }\end{array}$ & $\begin{array}{l}\text { 5'AAA TCA CAG GGC GTA GTT GTG'3 } \\
\text { 5'GAC CCA CCA GCC AAT CTT AG'3 }\end{array}$ & 556 & 58 & [24] \\
\hline blaKPC & $\begin{array}{l}\mathrm{KPC}(\mathrm{F}) \\
\mathrm{KPC}(\mathrm{R})\end{array}$ & $\begin{array}{l}\text { 5'CAGCTCATTCAAGGGCTTC' } 3 \\
\text { 5'AGTCATTTGCCGTGCCATAC'3 }\end{array}$ & 534 & 58 & [24] \\
\hline blaNDM-1 & $\begin{array}{l}\text { NDM-1 (F) } \\
\text { NDM-1 (R) }\end{array}$ & $\begin{array}{l}\text { 5'CTG AGC ACC GCA TTA GCC'3 } \\
\text { 5'GGG CCG TAT GAG TGA TTG C'3 }\end{array}$ & 754 & 60 & [23] \\
\hline
\end{tabular}

$\mathrm{CTX}=$ Cefotaxime, NDM=New Delhi metallo-beta-lactamase, $\mathrm{ESBL}=$ Extended spectrum beta lactamase

Table-2: Occurrence of ESBL producing isolates.

\begin{tabular}{|c|c|c|c|c|}
\hline \multirow[t]{2}{*}{ Site of specimen collection } & \multirow[t]{2}{*}{ Number of samples collected } & \multirow[t]{2}{*}{ Number of ESBL (\%) } & \multicolumn{2}{|c|}{$\begin{array}{c}\text { Number of ESBL } \\
\text { isolates }(\%)\end{array}$} \\
\hline & & & K. pneumoniae & E. coli \\
\hline Throat & 18 & $12(66.6)$ & 05 & 07 \\
\hline Wound & 39 & $08(20.5)$ & 03 & 05 \\
\hline Urine & 07 & $04(57.1)$ & 0 & 04 \\
\hline Environmental swabs & 18 & $06(33)$ & 05 & 01 \\
\hline Total & 82 & $30(36.6)$ & $13(15.8)$ & $17(20.7)$ \\
\hline
\end{tabular}

$\mathrm{ESBL}=$ Extended spectrum beta lactamase, $K$. pneumoniae=Klebsiella pneumoniae, $\mathrm{E}$. coli=Escherichia coli 


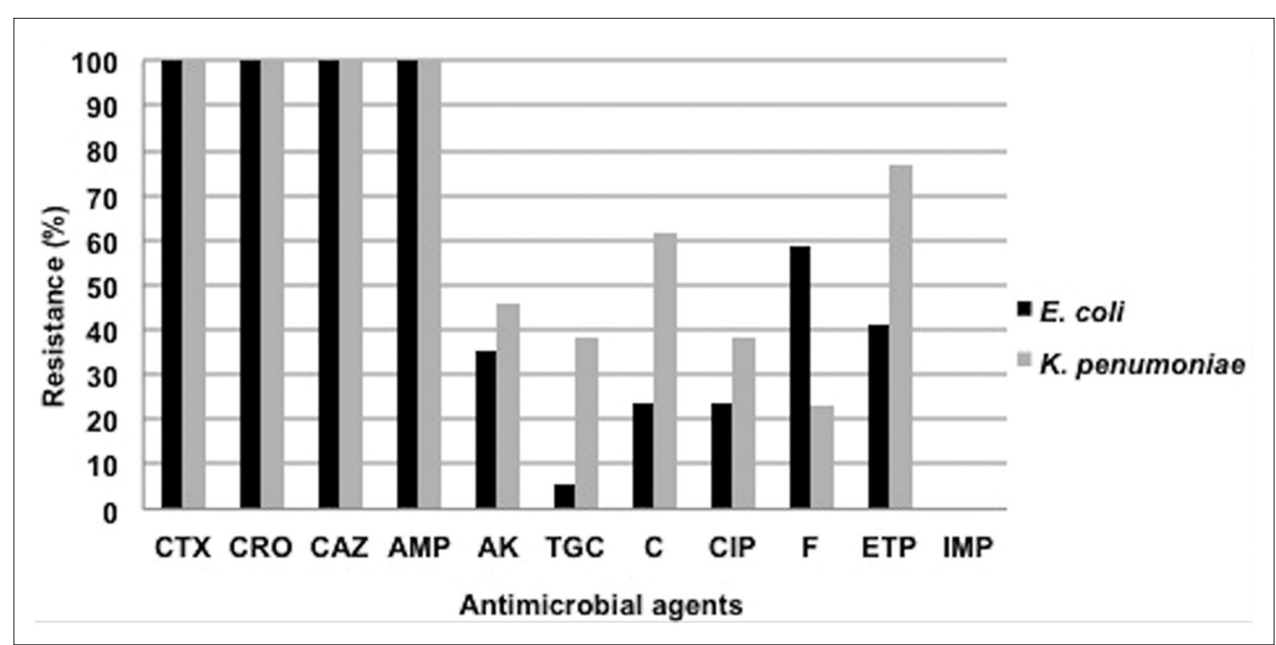

Figure-1: Percent resistance to different groups of antimicrobials among Klebsiella pneumoniae and Escherichia coli isolates. Cefotaxime (CTX), ceftriaxone (CRO), ceftazidime (CAZ), ampicillin (AMP), amikacin (AK), tigecycline (TGC), chloramphenicol (C), ciprofloxacin (CIP), nitrofurantoin (F), ertapenem (ETP), imipenem (IMP).

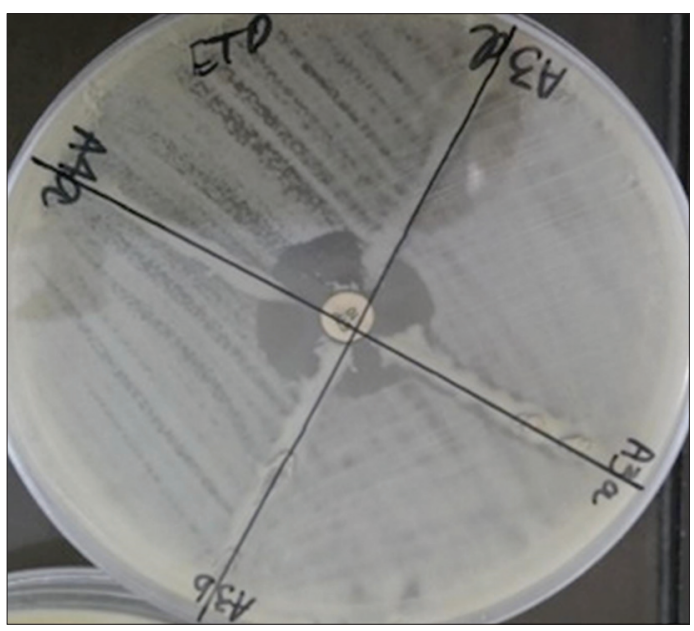

Figure-2: Modified Hodge test showing cloverleaf like indentation within the zone of inhibition of ertapenem disc.

blaTEM (Table-3). Three isolates carried both blaCTX and blaTEM. None of the isolates was positive for blaSHV. CTX-M positive isolates were subjected for CTX-M grouping, as shown in Table-3, blaCTX-M-1 was the most common group 17/21 (80.9\%) followed by blaCTX-M-9 observed in 02/21 (9.5\%) ESBL isolates. ETP resistant but IMP sensitive E. coli and K. pneumonaie isolates were also tested for carbapenemase-encoding genes blaKPC, blaNDM-1, blaOXA-48 and blaVIM. However, none of the ETP resistance isolate was positive with carbapenemase genes. Hence, these findings suggest that most of the ESBL producing isolates belonged to CTX-M-1 group (Table-3).

\section{Discussion}

The $\beta$-lactam group of antibiotics is the most common drugs used for the treatment of Gramnegative bacteria, and the ESBL mediated resistance has become a global challenge in infection control. Despite the recent worldwide spread of ESBL in $K$. pneumoniae and E. coli isolates from hospital-acquired infections, their dissemination has been little studied in developing countries. Globally, the prevalence of ESBL producing K. pneumonia and E. coli varies across different geographical areas with highest in Latin America (44\% and 13.5\%), followed by Asia/ Pacific $(22.4 \%$ and $12 \%)$, Europe $(13.3 \%$ and $7.6 \%)$, and North America (7.5\% and 2.2\%) [25]. In particular, incidence of ICU-acquired infections in developing countries is very high and accounted (pooled density 47.9 per 1000 patient-days) in contrast to the rate in the USA (13.6 per 1000 patient-days) [2].

In this study, we determined the prevalence and molecular classes of ESBL particularly of CTX-M type from ICU associated patients and inanimate environment from tertiary care hospitals in Faisalabad region of Pakistan. High incidence of ESBL producing K. pneumoniae and E. coli has been reported from clinical isolates of feces, urine, and wound infections in few studies in Pakistan [26,27]. Recently, the prevalence of ESBL producing K. pneumoniae and E. coli from UTIs reported to be $24.5 \%$ and $53.4 \%$, respectively [28]. In our study, overall ESBL phenotypes were observed in $15.8 \% \mathrm{~K}$. pneumoniae and $20.7 \%$ E. coli. This relatively low frequency could be due to low numbers however large diversity of samples compared to the previous report that focused only on isolates recovered from UTIs. However, in our study, the prevalence of ESBL producing $E$. coli from UTI (57.1\%) were almost similar to recent study [28]. In a previous study from tertiary care hospital from Pakistani, ESBL were found in $36 \% \mathrm{~K}$. pneumoniae and $41 \%$ E. coli [29]. However, these studies were based on phenotypic identification and did not involve molecular identification and characterization of ESBL genotypes. In this study, we also determined the ESBL producing bacteria from hospital inanimate environment such as devices, catheters, doors, and other fomites. None of the previous studies in Pakistan detected ESBL from hospital environment.

Since 2000, CTX-M type of ESBL has become clinically most important ESBL type due to its associated 
Available at www.onehealthjournal.org/Vol.2/10.pdf

Table-3: Molecular characterization of bla genes among ESBL-producing $K$. pneumoniae and $E$. coli isolates.

\begin{tabular}{lccccccc}
\hline ESBL isolates & \multicolumn{3}{c}{ ESBL genotype } & & \multicolumn{3}{c}{ CTX-M groups } \\
\cline { 2 - 3 } & CTX-M & TEM & SHV & & CTX-M-1 & CTX-M-2 & CTX-M-9 \\
\hline K. pneumonia $(n=10)$ & 9 & 3 & 0 & 7 & 10 & 0 & 2 \\
E. coli $(n=13)$ & 10 & 4 & 0 & & 0 & 0 \\
Total $(n=23)(76.6 \%)$ & $19(82 \%)$ & $7(30 \%)$ & 0 & & $17(80 \%)$ & 0 & $2(9.5 \%)$ \\
\hline
\end{tabular}

$\mathrm{ESBL}=$ Extended spectrum beta lactamase, $K$. pneumoniae=Klebsiella pneumoniae, $E$. coli=Escherichia coli, $\mathrm{CTX}=$ Cefotaxime

with MDR in many parts of the world including Africa, South America, Asia and Europe [11]. One particular example is pandemic spread of CTX-M-15 belong to CTX-M-1 group in nosocomial and community settings worldwide. There is lack of knowledge on the prevalence of clinical important CTX-M groups in ICU settings in Pakistan. This study showed very high carriage of CTX-M-1 group (80\%) in K. pneumoniae and E. coli. This is in agreement with previous findings globally and locally. Few earlier reports from Pakistan showed CTXM-1 as the most common ESBL instead of TEM and SHV. Previous findings reported $93.84 \%(\mathrm{n}=61)$ isolates of ESBL producing K. pneumoniae from a tertiary care hospital in Karachi, Pakistan [30]. In previous studies, all the ESBL producing $K$. pneumoniae and E. coli harbored CTX-M group-1 [26,31]. One particular concern is the association of CTX-M-1 producing bacteria with MDR [9]. Our data endorsed the fact that all the CTX-M positive strains were also MDR.

Although carbapenems are costly antibiotics, nevertheless sales in Egypt, India, and Pakistan have dramatically increased with better sensitivity and over the counter availability [2]. Carbapenem resistance has been rarely reported from Pakistan. This could be due to a large number of studies did not investigate carbapenemase encoding genes at molecular level. One study reported high prevalence (18.5\%) of E. coli producing carbapenemase of blaNDM-1 type in a military hospital in Rawalpindi, Pakistan. In our findings, we determined carbapenem resistance both phenotypically and genetically. All the isolates were sensitive to IMP which is in comparison to the recent study [28]. Moreover, none of the K. pneumoniae and E. coli found PCR-positive with carbapenemase-encoding genes. However, we found a high frequency of ETP resistant $K$. pneumoniae and $E$. coli isolates. These isolates could not show resistance against IMP and were also negative with PCR for any of the carbapenem genes studied. There is limited clinical data regarding ETP resistance in developing countries, and we found no report from Pakistan. In a previous study, ETP has been reported as a weak indicator for the detection of carbapenem resistance [32]. This is plausible because ETP resistance could be due to outer membrane porin loss [33].

\section{Conclusion}

This study shows large dissemination of MDR CTX-M-I producing $K$. pneumoniae and E. coli in patients and environment of ICUs in Pakistan.
Appropriate infection control measures should focus on reducing the spread of antibiotic resistance in ICU environment. We conclude that beside person-to-person transmission, ICU's environment could be a reservoir of MDR ESBL strains.

\section{Authors' Contributions}

AAB: Sample collection and bacterial cultivation. MIA: Sample collection and biochemical confirmations. SR: PCR and gel electrophoresis. MA: DNA extraction and quantification. SUR: Concept, article writing and revision. MM: Study design, writing and optimization of standards.

\section{Acknowledgments}

Higher Education Commission, Pakistan supported this research with Startup grant No IPFP/ HRD/HEC/2014. The project was partially funded by National Academy of Sciences (NAS), United States to Dr. Muhammad Imran Arshad under "One Health program".

\section{Competing Interests}

The authors declare that they have no competing interests.

\section{References}

1. Odonkor ST, Addo KK. Bacteria resistance to antibiotics: Recent trends and challenges. Int J Biol Med Res 2011;2:1204-10.

2. Laxminarayan R, Duse A, Wattal C, Zaidi AK, Wertheim HF, Sumpradit N. Antibiotic resistance-the need for global solutions. Lancet Infect Dis 2013;13:1057-98.

3. Flores-González JC, Hernández-González A, RodríguezLópez C, Roldán-Cano V, Rubio-Quiñones F, QuinteroOtero $\mathrm{S}$, et al. Nosocomial urinary tract infection in critical pediatric patients. Med Intensiva 2011;35:344-8.

4. Chong Y, Ito Y, Kamimura T. Genetic evolution and clinical impact in extended-spectrum ß-lactamase-producing Escherichia coli and Klebsiella pneumoniae. Infect Genet Evol 2011;11:1499-504.

5. Wu G, Day MJ, Mafura MT, Nunez-Garcia J, Fenner JJ, Sharma M, et al. Comparative analysis of ESBL-positive Escherichia coli isolates from animals and humans from the UK, The Netherlands and Germany. PLoS One 2013;8:e75392.

6. Gupta A, Ampofo K, Rubenstein D, Saiman L. Extended spectrum beta-lactamase-producing Klebsiella pneumoniae infections: A review of the literature. J Perinatol 2002;23:439-43.

7. Paterson DL, Bonomo RA. Extended-spectrum beta-lactamases: A clinical update. Clin Microbiol Rev 2005; 18:657-86

8. Rawat D, Nair D. Extended-spectrum ß-lactamases in gram 
negative bacteria. J Glob Infect Dis 2010;2:263-74.

9. Ruppé E. Epidemiology of extended-spectrum-beta lactamses: Rise of the CTX-M. Antibiotiques 2010;12:3-16.

10. Amos GC, Hawkey PM, Gaze WH, Wellington EM. Waste water effluent contributes to the dissemination of CTX-M15 in the natural environment. J Antimicrob Chemother 2014;69:1785-91.

11. Pitout JD, Laupland KB. Extended-spectrum beta-lactamase-producing Enterobacteriaceae: An emerging public-health concern. Lancet Infect Dis 2008;8:159-66.

12. Ewers C, Bethe A, Semmler T, Guenther S, Wieler LH. Extended-spectrum $B$-lactamase-producing and AmpCproducing Escherichia coli from livestock and companion animals, and their putative impact on public health: A global perspective. Clin Microbiol Infect 2012;18:646-55.

13. Cui L, Zhao J, Lu J. Molecular characteristics of extended spectrum B-lactamase and carbapenemase genes carried by carbapenem-resistant Enterobacter cloacae in a Chinese university hospital. Turk J Med Sci 2015;45:1321-8.

14. Kumarasamy KK, Toleman MA, Walsh TR, Bagaria J, Butt F, Balakrishnan R, et al. Emergence of a new antibiotic resistance mechanism in India, Pakistan, and the UK: A molecular, biological, and epidemiological study. Lancet Infect Dis 2010;10:597-602.

15. Poirel L, Pitout JD, Nordmann P. Carbapenemases: Molecular diversity and clinical consequences. Future Microbiol 2007;2:501-12.

16. Clinical and Laboratory Standards Institute. Performance Standards for Antimicrobial Susceptibility Testing; Twentieth Informational Supplement M100-S20. Wayne, PA. Clinical and Laboratory Standards Institute; 2010.

17. Sultan BA, Khan E, Hussain F, Nasir A, Irfan S. Effectiveness of modified Hodge test to detect NDM-1 carbapenemases: An experience from Pakistan. J Pak Med Assoc 2013;63:955-60.

18. Sambrook J, Fritsche E, Maniatis T. Molecular Cloning: A Laboratory Manual. $2^{\text {nd }}$ ed. Cold Spring Harbor, New York: Cold Spring Harbor Laboratory Press; 1989.

19. Cullik A, Pfeifer Y, Prager R, von Baum H, Witte W. A novel IS26 structure surrounds blaCTX-M genes in different plasmids from German clinical Escherichia coli isolates. J Med Microbiol 2010;59:580-7.

20. Lewis JS, Herrera M, Wickes B, Patterson JE, Jorgensen JH. First report of the emergence of CTX-M-type extended-spectrum $\beta$-lactamases (ESBLs) as the predominant ESBL isolated in a U.S. Health care system. Antimicrob Agents Chemother 2007;51:4015-21.

21. Tofteland S, Haldorsen B, Dahl KH, Simonsen GS, Steinbakk M, Walsh TR, et al. Effects of phenotype and genotype on methods for detection of extended-spectrum- $\beta$-lactamase-producing clinical isolates of Escherichia coli and Klebsiella pneumoniae in Norway. J Clinic Microbiol
2007;45:199-205.

22. Xu L, Ensor V, Gossain S, Nye K, Hawkey P. Rapid and simple detection of blaCTX-M genes by multiplex PCR assay. J Med Microbiol 2005;54:1183-7.

23. Pfeifer Y, Witte W, Holfelder M, Busch J, Nordmann P, Poirel L. NDM-1-producing Escherichia coli in Germany. Antimicrob Agents Chemother 2011;55:1318-9.

24. Gröbner S, Linke D, Schütz W, Fladerer C, Madlung J, Autenrieth IB, et al. Emergence of carbapenem-non-susceptible extended-spectrum beta-lactamase-producing Klebsiella pneumoniae isolates at the university hospital of Tübingen, Germany. J Med Microbiol 2009;58:912-22.

25. Falagas ME, Karageorgopoulos DE. Extended-spectrum beta-lactamase-producing organisms. J Hosp Infect 2009;73:345-54.

26. Habeeb MA, Haque A, Nematzadeh S, Iversen A, Giske CG. High prevalence of 16S rRNA methylase RmtB among CTX-M extended-spectrum B-lactamase-producing Klebsiella pneumoniae from Islamabad, Pakistan. Int J Antimicrob Agents 2013;41:524-6.

27. Mushtaq S, Irfan S, Sarma JB, Doumith M, Pike R, Pitout J, et al. Phylogenetic diversity of Escherichia coli strains producing NDM-type carbapenemases. J Antimicrob Chemother 2011;66:2002-5.

28. Ahmed I, Sajed M, Sultan A, Murtaza I, Yousaf S, Maqsood B. The erratic antibiotic susceptibility patterns of bacterial pathogens causing urinary tract infections. EXCLI J 2015; 14:916-25.

29. Jabeen K, Zafar A, Hasan R. Frequency and sensitivity pattern of extended spectrum beta lactamase producing isolates in a tertiary care hospital laboratory of Pakistan. J Pak Med Assoc 2005;55:436-9.

30. Khan E, Schneiders T, Zafar A, Aziz E, Parekh A, Hasan R. Emergence of CTX-M Group 1-ESBL producing Klebsiella pneumonia from a tertiary care centre in Karachi, Pakistan. J Infect Dev Ctries 2010;4:472-6.

31. Habeeb MA, Haque A, Iversen A, Giske CG. Occurrence of virulence genes, $16 \mathrm{~S}$ rRNA methylases, and plasmid-mediated quinolone resistance genes in CTX-M-producing Escherichia coli from Pakistan. Eur J Clin Microbiol Infect Dis 2014;33:399-409.

32. Tängdén T, Adler M, Cars O, Sandegren L, Löwdin E. Frequent emergence of porin-deficient subpopulations with reduced carbapenem susceptibility in ESBL-producing Escherichia coli during exposure to ertapenem in an in vitro pharmacokinetic model. J Antimicrob Chemother 2013;68:1319-26.

33. Carvalhaes CG, Picão RC, Nicoletti AG, Xavier DE, Gales AC. Cloverleaf test (modified Hodge test) for detecting carbapenemase production in Klebsiella pneumoniae: Be aware of false positive results. J Antimicrob Chemother 2010;65:249-51. 